\title{
Electron cyclotron heating and diagnostics of plasma at the second harmonic in the GDT device
}

\author{
E.D. Gospodchikov ${ }^{1,2}$, O.B. Smolyakova ${ }^{1,2}$, A.L. Solomakhin ${ }^{2}$, A.G. Shalashov ${ }^{1,2}$ \\ ${ }^{1}$ Institute of Applied Physics RAS, Nizhniy Novgorod, Russia, egos@appl.sci-nnov.ru \\ ${ }^{2}$ Budker Institute of Nuclear Physics SB RAS, Novosibirsk, Russia
}

\section{Introduction}

The electron cyclotron resonance heating (ECRH), being a standard technique for tokamaks and stellarators, has also good potentials for fusion-relevant experiments in large-scale open traps. In particular, efficient ECRH has been demonstrated recently in the mirror device GDT (gas-dynamic trap) in the Budker Institute [1-3]. In reported experiments, a combined plasma heating by neutral beams and microwave radiation results in the record electron temperatures up to $1 \mathrm{keV}$ at density about $10^{13} \mathrm{~cm}^{-3}$ in the regime with a narrow rf-power deposition profile and up to $350 \mathrm{eV}$ in the regime with a broad power deposition profile [1]. Success in ECRH experiments at GDT is convincing enough to consider the prospects of simple axially symmetric open magnetic traps as a high-power neutron source for fusion applications, including material testing, a hybrid fusion-fission reactor and nuclear waste processing [4].

Presently, the ECRH system in GDT is operating with the extraordinary mode at the fundamental cyclotron harmonic (X1). Although successful ECRH experiments at GDT evidence for the reliability of such heating mechanism, it may face essential difficulties in the next-step devices operating at higher $\beta$ [5]. First difficulty is related to the very complicate plasma dielectric response near the first cyclotron harmonic that results in strong refraction [6]. Although this feature is used in the present ECRH scheme and direct ECE measurements validate our understanding of the involved physics [7, 8], next-step devices would probably require simpler solutions. Other weakness of the X1 scheme is related to the accessibility condition $\omega>\omega_{\mathrm{pe}}$ for microwaves. In open magnetic configurations, this condition requires the launching system to be placed near a magnetic mirror, what is often technically not possible. This will be critical for the next generation devices which operate at higher electron densities.

Heating with the extraordinary mode at the second harmonic (X2) is free of the mentioned drawbacks; however it requires high enough electron temperature in order to be effective. In this context, ECRH at the second harmonic is possible in combination with heating at the first harmonic providing the initial high electron temperature. In this paper we discuss physical basics and peculiarities of regime. We also discuss our plans for experimental validation of combined $\mathrm{X} 1+\mathrm{X} 2 \mathrm{ECRH}$ regime in GDT by measuring the electron cyclotron emission at the second harmonic (X2 ECE).

\section{ECRH at the second harmonic}

Basics physic of electron cyclotron heating at the second harmonic is well known [9]. In tokamak conditions, effective absorption requires quasi-transverse to the external magnetic field launch of a microwave beam with extraordinary wave polarization. Essentially the same condition remains in force in an open trap. However, here a new physics appear related to the variation of a strength of the magnetic field along its direction (presumably $\mathbf{B} \| \nabla B$ in open traps while $\mathbf{B} \perp \nabla B$ in toroidal systems). On one hand, one can assume that quasi-transverse direction corresponds to approximately constant magnetic field along propagation path of the microwave beam. But the longitudinal wave vector is not constant due to $\nabla B$ inhomo-geneity, thus a strong influence of the refraction is expected in an open magnetic configuration. In some sense this situation is similar to the "top launch" scenario for ECRH in tokamaks [10].

The resonance broadening in X2 ECRH is presumably defined by the relativistic electron mass,

$$
\left|\omega-2 \omega_{c e}\right| / \omega \sim T_{e} / m_{e} c^{2} \sim 10^{-3} .
$$

Having in mind that the electron temperature in open trap is noticeably lower than in modern toroidal systems, one can expect an extremely narrow relativistic resonance region in the real space. Because of this feature, a strictly transverse launch of a wave beam is ineffective. This is illustrated in figure 1. One can see that transverse propagation corresponds to high value of optical thickness for very small fraction of rays contributing the microwave beam of finite aperture. In this situation most part of the wave beam will not be absorbed, see 1(a). With more oblique propagation, the peak optical thickness reduces, but the fraction of absorbed rays is increased. The optimal total absorption is realized at the propagation angle for which the spread of the starting positions of the absorbed rays is close to the beam width, see 1(c).

In GDT conditions this corresponds to the launching angle $\theta=80^{\circ}$ (angle between the wave vector and the magnetic field) for straight ray approximation and $\theta=85^{\circ}$ for ray-tracing modeling. This launching direction is preferable for X2 ECE and ECRH experiments. For the optimal launching angle one can expect absorption efficiency $60-70 \%$ for $T_{\mathrm{e}}=500 \mathrm{eV}$ and $10-20 \%$ for $T_{\mathrm{e}}=150 \mathrm{eV}$.

\section{Second harmonic ECE of bulk and hot electrons}

As a first step aimed at experimental validation of combined $\mathrm{X} 1+\mathrm{X} 2 \mathrm{ECRH}$ regime in GDT we are going to measure the electron cyclotron emission in the extraordinary mode at the second harmonic (X2 ECE), what is obviously less expensive than implementing the $\mathrm{X} 2$ ECRH. The idea of the experiment is to exploit the reciprocity principle and Kirchhoff's law that link the absorption and the spontaneous plasma emission at thermal equilibrium. According to this idea, finding the X2 ECE with the effective radiation temperature comparable with 
the electron temperature would indicate in favor of efficient X2 ECRH in the geometry reversed to the ECE one. Such measurements will be performed with a new ECE diagnostics operating at $75 \mathrm{GHz}$ (under construction now) in high-temperature $\left(T_{\mathrm{e}} \sim 500 \mathrm{eV}\right)$ discharges supported by $54.5 \mathrm{GHz}$ ECRH at the fundamental harmonic. In addition, new ECE diagnostics will provide data on the lineintegrated electron pressure in discharge with a high resolution in time.

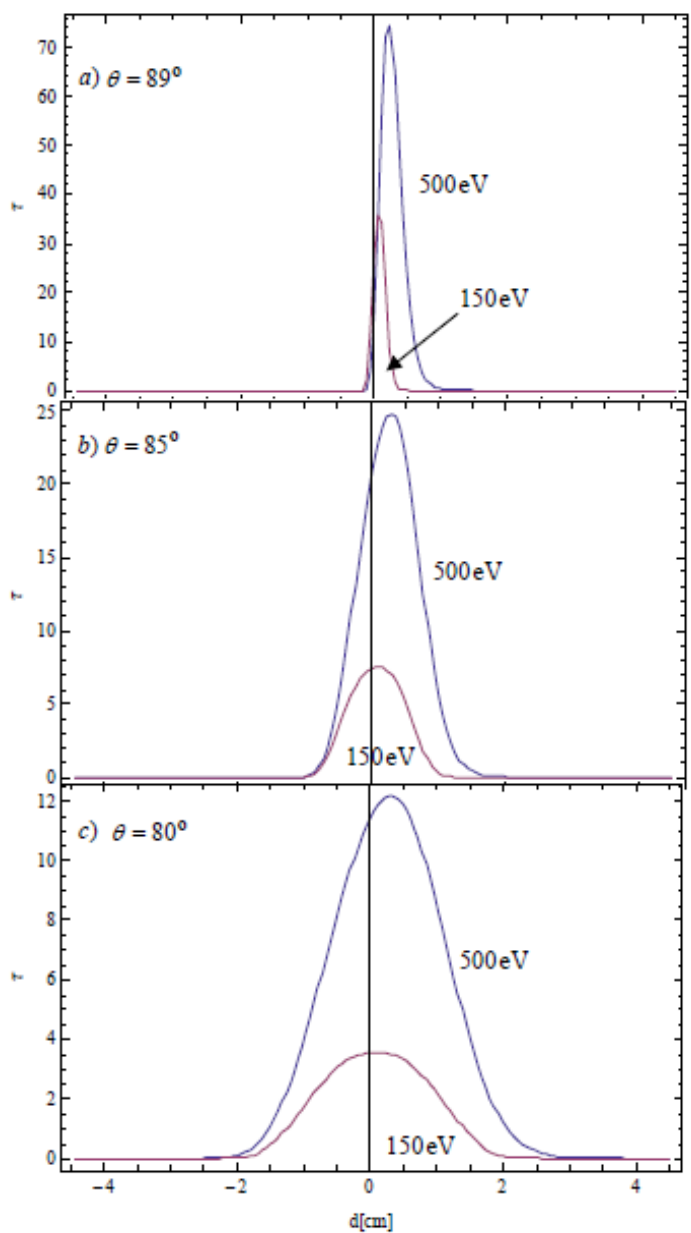

Fig. 1. Dependence of optical thickness $\tau=\int \operatorname{Im} k d l$ on transverse position $d$ of geometric-optics ray for electron temperatures $T_{\mathrm{e}}=150 \mathrm{eV}$ and $T_{\mathrm{e}}=500 \mathrm{eV}$. Here $d$ is shift from the central ray of microwave beam (crossing the resonance surface at a trap axis)

The similar idea was used previously to confirm our understanding of X1 ECRH at GDT with the ECE operating in the vicinity of the ECRH frequency $54.5 \mathrm{GHz}[7$, 8]. Besides, measured by this diagnostics non-thermal ECE have unambiguously confirmed the existence of suprathermal electrons in GDT with energies about 10 $\mathrm{keV}$. These electrons are generated by the intense microwave field during the ECR discharge start-up and heating of the main plasma [11]. Presence of suprathermal electrons should be taken into account in the analysis ECE at the second harmonic. Figure 2 illustrates possibility for separation of signal from hot $(10 \mathrm{keV})$ and thermal $\left(T_{\mathrm{e}}=500 \mathrm{eV}\right)$ electrons exploiting the relativistic shift of the resonance frequency. Here expected radiation temperatures are calculated for the antenna which is now installed in GDT chamber.

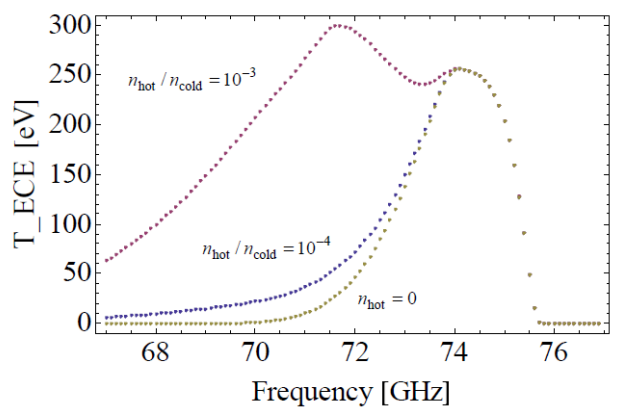

Fig. 2. Radiation temperature for different hot electron densities for 2X ECE experiments preparing at GDT

In conclusion we note that for relatively warm plasmas recently obtained at GDT $\left(T_{\mathrm{e}}=500 \mathrm{eV}\right)$ one can expect an efficient electron cyclotron heating at the second harmonic. For checking this concept we install new ECE system at $75 \mathrm{GHz}$. receiving system for registration of. Observation of near blackbody plasma emission at the second electron cyclotron harmonic should demonstrate perspective of similar heating scenarios in open magnetic traps.

The work was supported by the Russian Science Foundation (pr. 14-12-01007).

\section{References}

1. P.A. Bagryansky, A.G. Shalashov, E.D. Gospodchikov et al. Phys. Rev. Letters. 2015. V. 114. P. 205001.

2. P.A. Bagryansky, E.D. Gospodchikov, Yu.V. Kovalenko et al. Fusion Science and Tech. 2015. V. 68. P. 87.

3. P.A. Bagryansky, A.V. Anikeev, G. G. Denisov et al. Nuclear Fusion. 2015. V. 55. P. 053009.

4. A.A. Ivanov, V.V. Prikhodko. Plasma Phys. Control. .Fusion. 2013. V. 55. P. 063001.

5. V.V. Postupaev, A.V. Burdakov, A.A. Ivanov Fusion Science and Tech. 2015. V.68. No.1. P. 92.

6. A. Shalashov, E. Gospodchikov, O. Smolyakova et al. Physics of Plasmas. 2012. V. 19. P. 052503

7. A.L. Solomakhin et al. AIP Conf. Proc. 2016. V. 1771. P. 050001 .

8. A.G. Shalashov et al. Electron cyclotron emission at the fundamental harmonic in GDT magnetic mirror. arXiv:1611.09137.

9. M. Bornatici. Plasma Phys. 1982. V.24. P.629.

10. J.-P. Hogge, S. Alberti, L. Porte and G. Arnoux. Nuclear Fusion. 2003. V. 43, No.11. P. 1353.

11. D.V. Yakovlev, A.G. Shalashov, E.D. Gospodchikov et al. Nuclear Fusion. 2016. V. 57. P. 016033 